

\title{
MINGA: MODELO REPLICABLE DE RENOVACIÓN URBANA SOSTENIBLE, CASO BUENAVENTURA
}

\author{
MINGA: SUSTAINABLE AND REPLICABLE URBAN RENOVATION \\ MODEL, THE BUENAVENTURA CASE
}

\author{
VALERIA VILLAMIL CÁRDENAS \\ Estudiante de arquitectura \\ Pontificia Universidad Javeriana \\ Cali, Colombia \\ https://orcid.org/0000-0002-9016-2374 \\ villamilvaleria@javerianacali.edu.co
}

\author{
IVÁN OSUNA-MOTTA \\ Magister en Arquitectura y Urbanismo \\ Doctorante en Arquitectura y Urbanismo \\ Director de la Maestría en Hábitat Sustentable \\ Pontificia Universidad Javeriana \\ Cali, Colombia \\ https://orcid.org/0000-0001-5137-2603 \\ ivan.osuna@javerianacali.edu.co
}

\section{RESUMEN}

Este artículo presenta los resultados obtenidos en la investigación realizada durante la ejecución de un diseño de renovación urbana sostenible en la ciudad de Buenaventura, Valle del Cauca, Colombia, como parte de la propuesta del equipo MINGA para el SDLAC 2019 (Solar Decathlon Latin America and Caribbean). Este proyecto fue desarrollado por un grupo de estudiantes y profesores, dentro de los espacios académicos de los programas de pregrado de Arquitectura e Ingeniería Civil de las universidades partícipes del equipo MINGA. Se utilizó la metodología de enseñanza-aprendizaje basada en proyectos, con integración curricular en cursos interdisciplinares tipo taller de proyectos. El objetivo principal fue demostrar la viabilidad de un proyecto de urbanismo resiliente, concebido para el clima futuro en una ciudad costera del trópico cálido-húmedo. Los resultados demostraron que se puede crear un urbanismo climático, resiliente al clima, que garantice la permanencia de los habitantes originales de las zonas costeras, mitigando los riesgos por inundación y garantizando el arraigo cultural de sus habitantes, aun en escenarios de aumento en el nivel del mar

Palabras clave

urbanismo sustentable, cambio climático, vivienda social

\section{ABSTRACT}

This article presents the results obtained in research made during a sustainable urban renewal design in the city of Buenaventura, Valle del Cauca, Colombia, as part of MINGA team's proposal for SDLAC 2019 (Solar Decathlon Latin America and Caribbean). This project was developed by a group of students and professors, as part of the undergraduate programs of architecture and civil engineering of the partner universities in the MINGA team. A project-based teaching-learning methodology was used, integrating the curricula in interdisciplinary project workshop-type courses. The main goal was to demonstrate the viability of a resilient urban planning project, conceived for the future climate in a coastal city in the hothumid tropics. The results showed that a climate-resilient urbanism can be created, which guarantees the permanence of the original inhabitants of the coastal areas, mitigating flooding risks, and preserving the cultural roots of the inhabitants, even under sea-rise scenarios. 


\section{INTRODUCCIÓN}

El Solar Decathlon es actualmente el evento académico de construcción sostenible más importante a nivel global; se desarrolla desde 2002 y declara dentro de sus objetivos la educación de estudiantes y público respecto a los beneficios ambientales que presenta la construcción sostenible (Kos y De Souza, 2014). Las ediciones latinoamericanas del concurso, realizadas en Cali, son pioneras al enfocar sus esfuerzos en las soluciones de vivienda sostenibles para poblaciones de bajos recursos, con relevancia regional para el trópico (Herrera-Limones, León-Rodríguez y LópezEscamilla, 2019). Para la edición 2019, se realizó una alianza entre la Pontificia Universidad Javeriana Cali (Colombia), la Universidad Federal de Santa Catarina y el Instituto Federal de Santa Catarina (Brasil), que derivó en la conformación de un equipo interdisciplinario constituido por estudiantes y profesores de arquitectura, ingenierías, diseño de comunicación visual y comunicación. El equipo Minga, diseñó, construyó y puso en funcionamiento un prototipo de vivienda que hace parte de un proyecto habitacional multifamiliar urbano, para la ciudad de Buenaventura. Si bien la propuesta urbana se sitúa en un lugar específico, teniendo en cuenta las condiciones medioambientales, sociales y económicas de la ciudad y la región, con un proyecto que responde a las necesidades de la población, es adaptable a otros territorios con condiciones similares de costa en el trópico cálido-húmedo.

Las áreas urbanas en zonas costeras del planeta son las más vulnerables a los efectos negativos del cambio climático, por estar muy pobladas y tener una mayor proyección de crecimiento poblacional (Béné et al., 2018; Neumann, Vafeidis, Zimmermann y Nicholls, 2015). En estas regiones y particularmente en Latinoamérica, existen altos índices de pobreza, exclusión, inequidad y precariedad de las viviendas, que convierten a la vida en las costas en una opción de alto riesgo (Nicholls et al., 2014; Vergel Tovar, 2010). Todo esto plantea nuevos desafíos para las ciudades costeras: hacerlas más viables y apropiadas para las condiciones climáticas futuras, priorizando la protección de la vida de sus habitantes y la protección de las infraestructuras frente a los efectos negativos del cambio climático, mediante estrategias de adaptación que se anticipen a los eventos y reduzcan la vulnerabilidad (Hernández-Guerrero, Vieyra-Medrano y Mendoza, 2012).

Ante los nuevos desafíos se hacen necesarias aproximaciones metodológicas capaces de desarrollar modelos urbanos climate-proof (Wardekker, De Jong, Knoop y Van Der Sluijs, 2010) orientados hacia el urbanismo climático como un nuevo paradigma que supera el concepto de urbanismo sostenible (Long y Rice, 2019). Es en este escenario de cambio que cobra mayor importancia el concepto de resiliencia urbana, entendida como la capacidad de un sistema para mantener o regresar rápidamente a su funcionamiento deseado, luego de una perturbación (Meerow, Newell y Stults, 2016). Se requiere que los sistemas urbanos incrementen su capacidad de adaptación, anticipando una respuesta adecuada a los desafíos actuales y futuros (Hernantes, Maraña, Giménez, Sarriegi y Labaka, 2019).

El caso de estudio plantea además el desafío de actuar sobre la ciudad informal. Tradicionalmente, los grandes proyectos urbanos dan respuesta a un entorno físico que precisa transformarse, pero en muy pocas ocasiones se dirigen a las verdaderas necesidades de sus pobladores (HernándezAraque, 2016). Se debe, así, desarrollar procesos de diseño, con participación comunitaria, que ofrezcan respuestas "situadas", relacionadas adecuadamente con contextos específicos (Musango, Currie, Smit y Kovacic, 2020). La propuesta aquí expuesta plantea que es posible elaborar un modelo de renovación urbana resiliente, que minimice el desplazamiento forzado dentro de la ciudad y desacelere la expansión del área urbana, mediante un proyecto que considere las condiciones climáticas actuales y futuras, las condiciones socioculturales y que reinterprete los sistemas de ocupación del territorio ancestrales, con el uso de nuevas tecnologías de construcción y procesos de diseño innovadores.

\section{CONTEXTUALIZACIÓN}

Buenaventura es una ciudad costera del suroccidente colombiano ( $3^{\circ} 52^{\prime} 59^{\prime \prime}$ Norte, $77^{\circ} 4^{\prime} 1^{\prime \prime}$ Oeste). Posee uno de los puertos marítimos más importantes del país debido a su posición estratégica, su conexión con el Océano Pacífico y cercanía con el canal de Panamá. La ciudad está dividida en dos: la parte continental y la isla de Cascajal, conectadas entre sí a través de la vía principal de la ciudad, la avenida Simón Bolívar. Asimismo, dicha relación con el mar, resulta un aspecto muy importante para la economía, la riqueza ambiental y la cultura de la ciudad. A pesar de esto, por razones de índole político, los ingresos económicos no se ven reflejados en la infraestructura de la ciudad; es por esto que las condiciones de vida de los habitantes son, en muchos casos, precarias.

De acuerdo a la clasificación climática de Köppen-Geiger, los climas de Colombia son tipo " $\mathrm{A}$ " que corresponden a los tropicales húmedos. Específicamente, Buenaventura posee un clima de selva tropical "Af", que presenta altas temperaturas (alrededor de $30^{\circ} \mathrm{C}$ ), con variaciones muy pequeñas durante el año, abundantes precipitaciones (150$1000 \mathrm{~cm}$ ), bastante nubosidad y una alta humedad (Rafferty, 2009). Gracias a su ubicación, la ciudad cuenta con una gran diversidad y riqueza natural. Aunque posee una importante cantidad de especies de flora y fauna, a nivel urbano carece de espacios con adecuadas condiciones ambientales. Se han proyectado áreas de expansión urbana en el Plan de Ordenamiento Territorial - POT (Alcaldía de Buenaventura, 2001) que perpetúan la deforestación y contempla el uso de muchos ecosistemas importantes para tal fin, como los manglares (Figura 1).

Con una población estimada de 440.995 habitantes (DANE, 2005), contando tanto el área rural como la urbana, las 

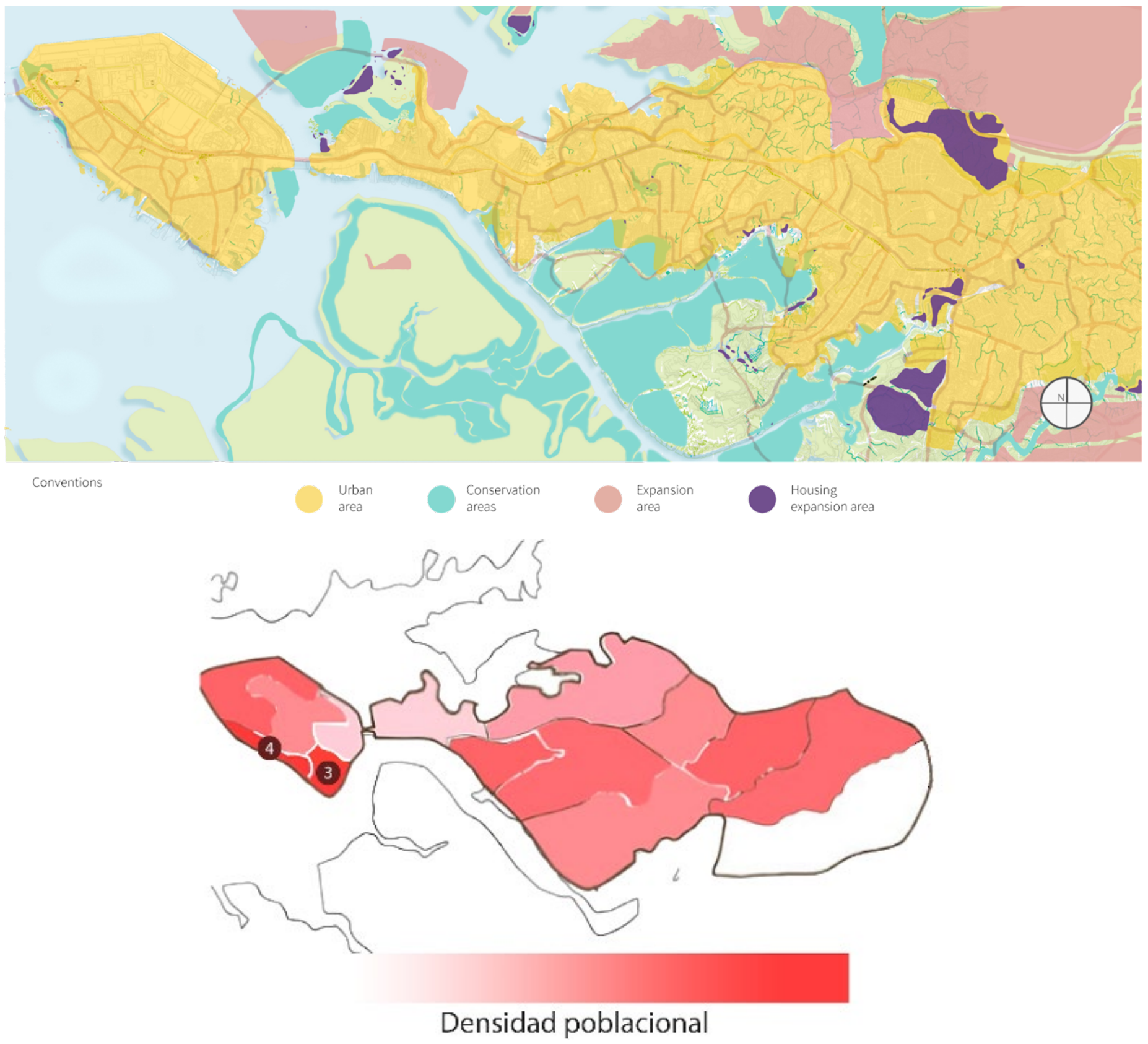

Figura 1. Zonas de expansión / Densidad Poblacional de Buenaventura. Fuente: Elaborado por los autores

comunas (zonas) con mayor densidad se encuentran hacia el borde sur de la isla (especialmente las 3 y 4) (Figura 1), donde se asientan de manera informal y en condiciones de hacinamiento debido a la gran cantidad de personas por vivienda, en pocos metros cuadrados. En ese sector de la isla se concentra la mayoría de hogares con necesidades Básicas Insatisfechas (NBI) y miseria del municipio (DANE, 2020). Efectivamente, en Buenaventura, más del 15\% de la población no cuenta con los servicios básicos (DANE, 2018) y las áreas mencionadas están dentro de las más afectadas en ese sentido. Además, los niveles de violencia son mayores en estas comunas respecto al resto de la ciudad.

Sumado a lo anterior, diversas problemáticas sociales y económicas impactan a la comunidad, entre ellas: el desplazamiento forzado, la falta de equidad y de oportunidades laborales, el poco acceso a la educación y a la salud, el analfabetismo, ilegalidad, etc. (Martínez et al., 2013). Tan solo en el año 2013 fueron desplazadas más de 13.000 personas de manera forzosa a la ciudad (Schoening, 2014), lo cual puede explicar el creciente desarrollo de asentamientos informales, sobretodo en el borde de la isla, que se han extendido hacia el mar con condiciones precarias y carencia de recursos para su habitabilidad

Es así como, hoy en día, los bordes de la isla de Cascajal están compuestos mayoritariamente de relleno utilizado por los pobladores con el fin de ganar terreno hacia el mar, expandiendo sus los límites urbanos. Para ello, se usa un sistema de construcción tradicional: la palafítica (Figura 2). 

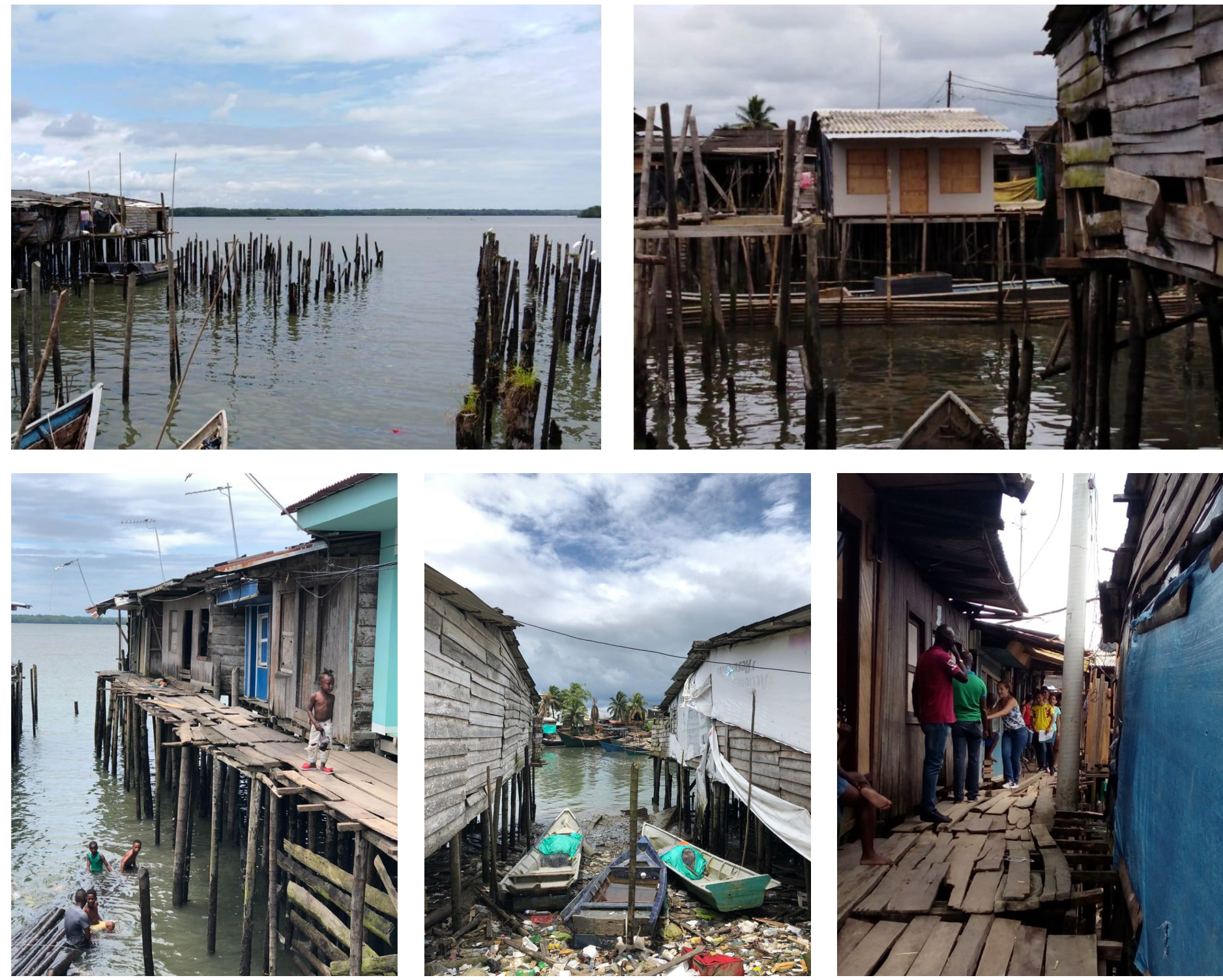

Figura 2. Estructuras existentes en la zona de estudio. Fuente: Elaborada por los autores

En ella, se hincan los palafitos (palos de palma de chonta) en el lecho marino, para fijar una base hecha de la misma madera y construir sobre esta, rellenando después debajo con material de residuo. Esto causa una alta contaminación del mar y pone en peligro a las personas que allí se asientan debido al riesgo de inundación existente en gran parte de la zona sur de la isla y al oeste del área continental (Figura 3). Asimismo, la falta de planeación ha generado deficiencia o ausencia de sistemas de acueducto y alcantarillado, entre otros sistemas deficientes o inexistentes.

Casos similares se han estudiado a nivel regional, como en Morelia (México), ya que se pueden asociar los eventos de inundación con la manera cómo se concibe la ciudad. La falta de planeación de la misma y de una respuesta acertada por parte de las autoridades hace evidente que las diferencias socioeconómicas respecto a quienes habitan en la periferia (como es también el caso de la comunidad bonaverense) refleja una notoria "inequidad frente al riesgo"; por esto la noción de adaptabilidad que se pretende exponer a partir del proyecto MINGA y el caso de estudio en Morelia, se convierte en una especie de eufemismo de "justicia social" (Hernández-Guerrero et al., 2012).

La Organización Mundial de la Salud afirma que los espacios públicos "verdes" y "azules" pueden mejorar en gran medida la salud, física y mental, y la calidad de vida de las personas, reduciendo los niveles de estrés, comorbilidades y propiciando espacios de descanso, recreación y actividad física (World Health Organization, 2016). Actualmente, Buenaventura tiene un índice de espacio público de $0.51 \mathrm{~m}^{2}$ por habitante, representado en la carencia parques públicos, muy por debajo de las ciudades que fueron objeto del estudio ya mencionado. Por otra parte, respecto al sistema vial, la avenida Simón Bolívar es un eje que comunica la ciudad con el resto del país. Está proyectada una vía en el lado norte de la isla que será construida con un objetivo más comercial y de transporte pesado, lo que dinamizará esta zona de la ciudad y consolidará el sur como una zona residencial 

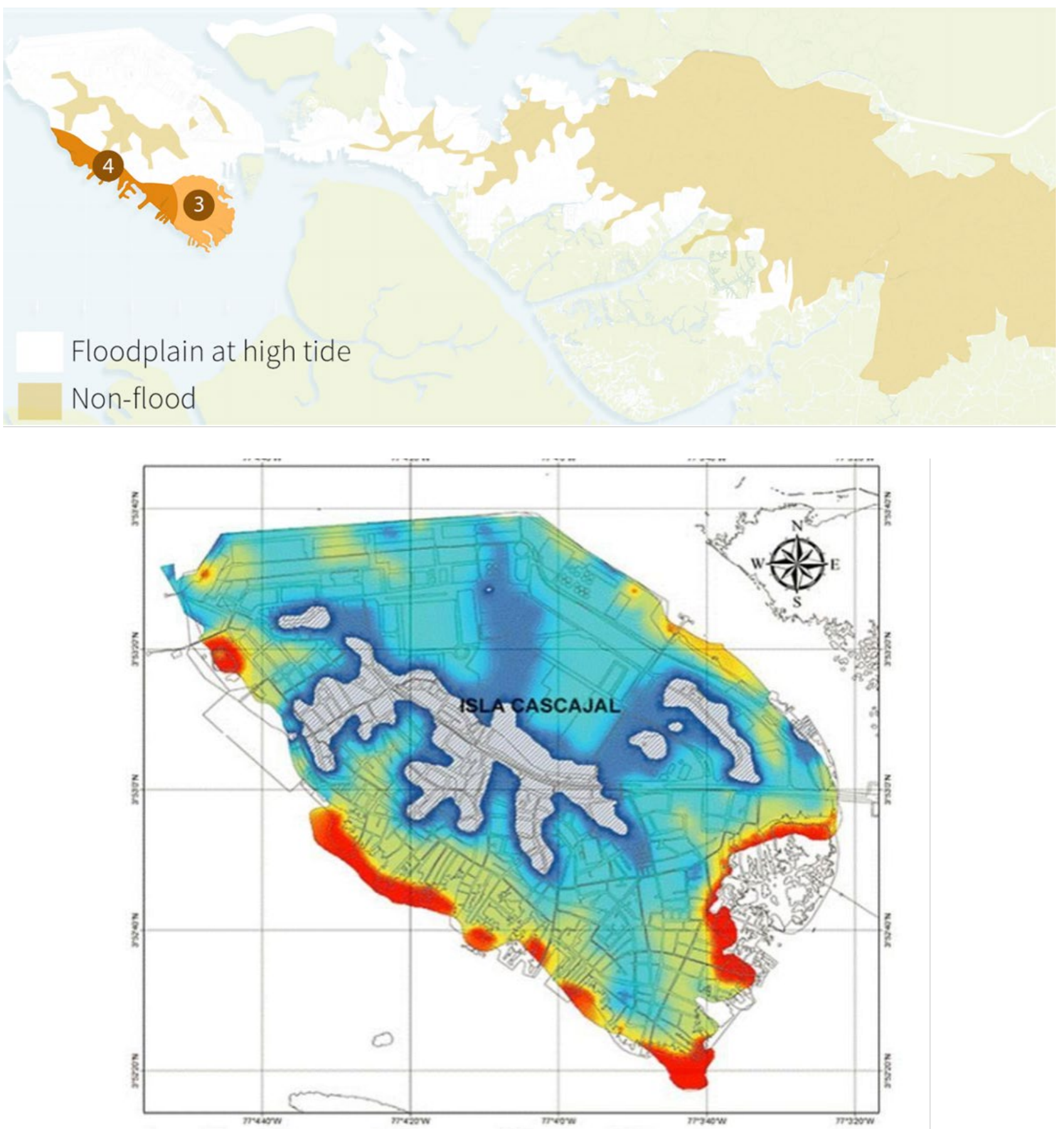

Figura 3. Mapa de inundación de Buenaventura y la Isla de Cascajal. Fuente: superior: Elaborada por autores. Inferior: Cocuñame y Salcedo (2017, p. 200).

y de transporte liviano. Así, el equipo MINGA entiende las variables del contexto y toma la decisión de trabajar principalmente el borde de la isla de Cascajal y un sector del borde del área continental.

\section{METODOLOGÍA}

En atención a los planteamientos del concurso, la propuesta urbana de MINGA se realizó integrando las temáticas del Solar Decathlon en los cursos de últimos años de pregrado de las carreras de Arquitectura e
Ingeniería Civil de la Javeriana Cali y la UFSC, cursos en los cuales se implementó la metodología de enseñanzaaprendizaje basada en proyectos que permite a los estudiantes poner en práctica los conocimiento teóricos adquiridos en los cursos tradicionales (Herrera-Limones, Rey-Pérez, Hernández-Valencia y Roa-Fernández, 2020; Jin et al., 2018; Osuna-Motta, 2018). Con este enfoque, la investigación, realizada por más de 80 estudiantes y 12 profesores de Colombia y Brasil, se centró en la temática del diseño urbano en densidad media aplicado al contexto del trópico latinoamericano, para lograr una propuesta 

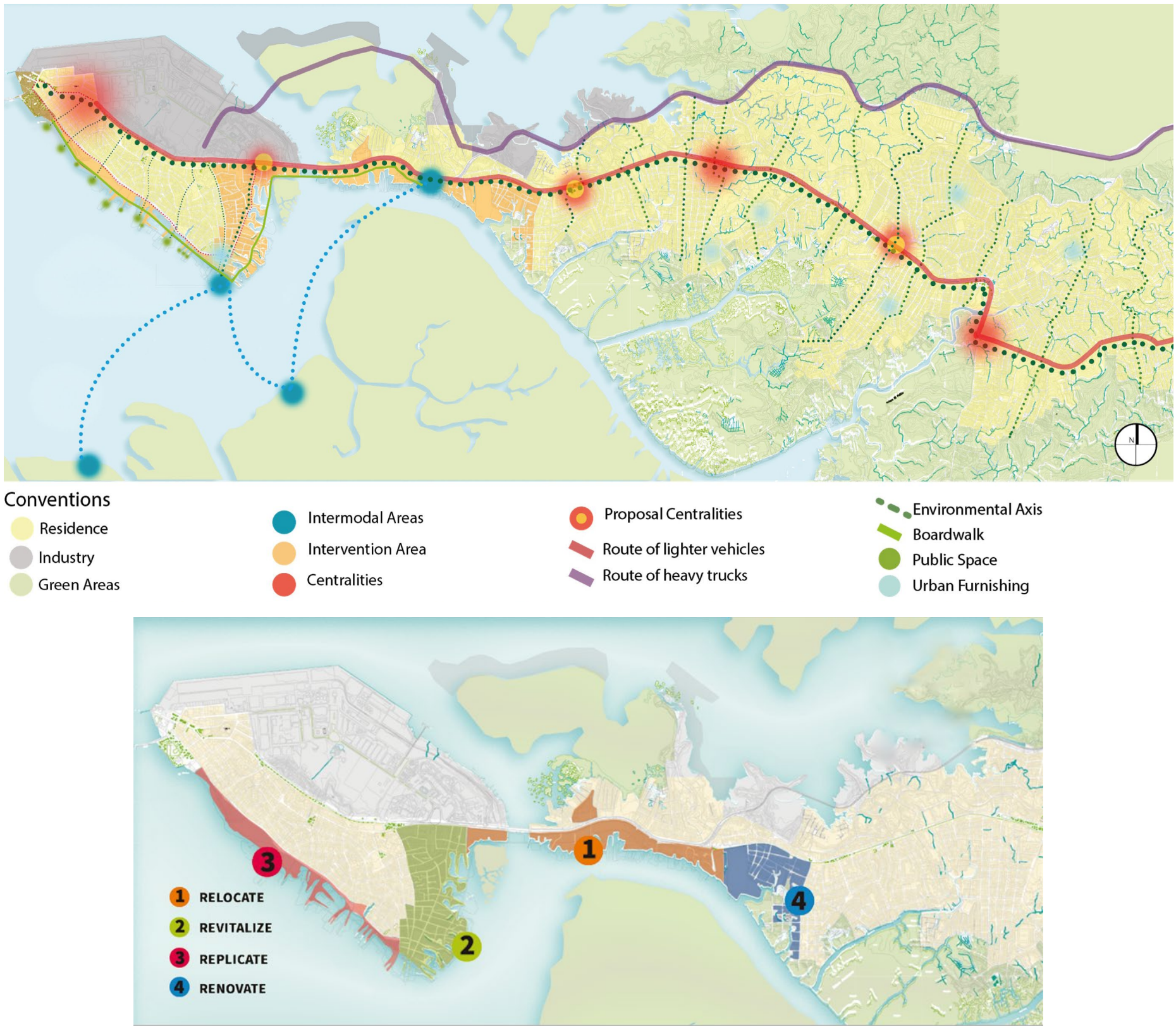

Figura 4. Plan Maestro Urbano. Propuesta del equipo MINGA 7 Fases del proyecto. Fuente: Elaborada por los autores

innovadora inspirada en viviendas sociales de bajo costo, sostenibles para las condiciones de clima en los próximos 50 años. El proceso fue el siguiente:

1. Se realizó un análisis e investigación del territorio, abarcando las condiciones medioambientales, históricas, sociales y económicas, viales, morfológicas, de los servicios públicos, de normativa, de usos y equipamientos y de riesgos.

2. Se inició con un esquema básico, yendo de una escala macro a una micro, identificando cuáles eran los lugares de la ciudad con más riesgos y condiciones críticas para trabajar sobre ellos dentro de un plan maestro.

3. En seguida, se planteó un Plan Maestro Urbano que comprendió tanto la zona continental como la isla de Cascajal, y contempló 3 fases donde se incorporó estrategias de relocalización de los habitantes de las zonas a afectar y diseño de vías y zonas a intervenir (tanto para desarrollo de vivienda como para equipamientos y espacio público). También se desarrolló a mayor detalle la escala de sitio, que abarca varias manzanas en el sector sur de la isla, replicando el prototipo de vivienda en las edificaciones en altura.

4. Finalmente, se realizó un prototipo de vivienda que fue construido a escala real para demostrar su funcionamiento y un modelo a escala 1:250 que muestra las unidades de vivienda que conforman las edificaciones en altura diseñadas para el plan urbano, además de algunas zonas de espacios públicos y la propuesta en cuanto a diseño vial. 


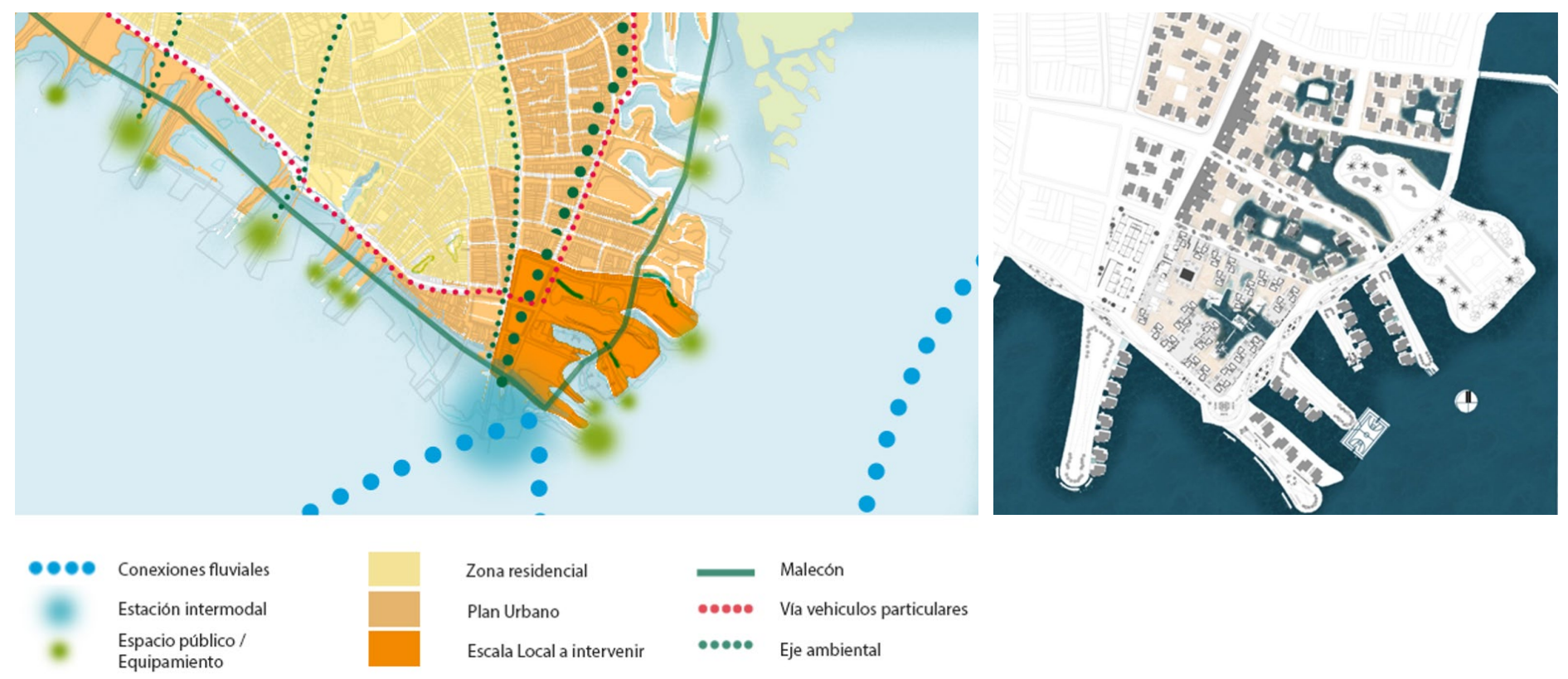

Figura 5. Localización y propuesta a escala. Fuente: Elaborada por los autores.

\section{RESULTADOS Y DISCUSIÓN}

Se desarrolló una propuesta de renovación urbana factible, en un sector para el cual las autoridades municipales han planteado un proceso de desalojo debido al alto riesgo de inundación existente en la zona. La medida, que no considera alternativas de mitigación para conservar a los habitantes en su lugar, generaría el desarraigo de una población que depende económica y culturalmente de su relación con el mar. A continuación, se explica el modelo a partir de las escalas en las que se trabajó: de macro a micro.

\section{PLAN MAESTRO URBANO}

Comenzando por la escala mayor, la propuesta urbana del equipo MINGA busca, a partir de la comprensión del contexto, generar estrategias para la solución a distintas problemáticas de la comunidad bonaverense. Una de las primeras consiste en generar dos vías importantes para la conexión entre la isla y la zona continental: una sería la ya propuesta por el POT, al norte de la ciudad, que recibiría el tráfico pesado y serviría como una conexión comercial para la ciudad. La otra vía sería el malecón -que se detallará más adelante-, diseñado para proporcionar el espacio público del que carece la ciudad y con varias opciones de trasporte limpio.

De la misma manera se recuperarían distintas rutas ya existentes para aprovecharlas como ejes ambientales: en primer lugar, creando un gran separador verde que recorra la avenida Simón Bolívar (av. SB), la cual cruza toda la ciudad. En segundo, se ubicarían varios puntos de conexión importantes a lo largo de esta avenida, con la idea de que se conviertan en ejes ambientales secundarios y rematen en espacios públicos y equipamientos de orden recreativo mayormente. En tercer término, paralelamente al malecón, se habilitaría la calle 1 ra que se deriva al oeste de la avenida Simón Bolívar al oriente de la isla, recorre gran parte del borde y se une con la carrera 20, la cual lleva al lado este de la avenida principal (Simón Bolívar). Este camino se dispondría para transporte liviano, otros medios de transporte ya usados como el vehículo particular, taxis, buses que ya circulan en la ciudad, etc. (Figura 4).

A escala local, el proyecto se desarrollaría en 4 fases (Figura 5):

1. Relocalización: en esta etapa se pretende conectar la isla con la zona continental de la ciudad, mientras se genera espacio público y comercial a lo largo del puente del Piñal, la única infraestructura vial que conecta la isla con el continente en la actualidad. A su vez, se reubicaría a la población del área intervenida en un principio, de manera que se relocalizarían temporalmente en la porción continental de la ciudad dando paso a la segunda fase.

2. Revitalización: se procuraría revitalizar la primera zona a intervenir en dos frentes: el público, con la realización del tramo correspondiente del malecón, junto con los equipamientos y espacios públicos proyectados; y el privado, con la construcción de las zonas residenciales.

3. Replicación: el malecón se conectaría con el ya existente y todo el modelo de urbanización sería replicado a lo largo de los bordes sur y oeste de la isla de Cascajal, uniéndola, a su vez, con el continente. Se trata esta de una acción detonadora que pueda propiciar y promover la correcta urbanización hacia el sur y la consolidación de esta zona residencial con el espacio público que requiere.

4. Renovación: esta última fase consolidaría el proyecto de renovación urbana, conectando el borde entero de la isla con la zona continental. 

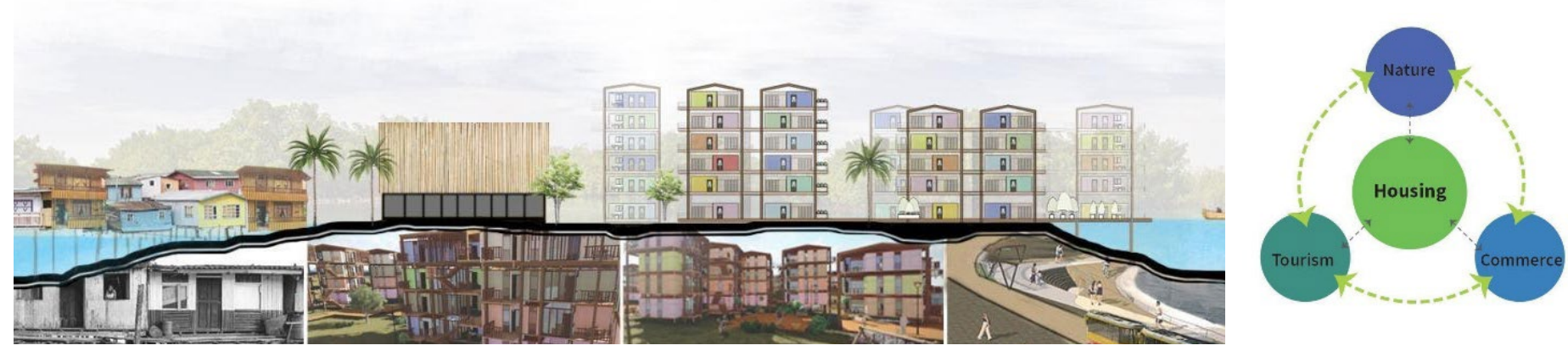

Figura 6. Corte urbano de la propuesta y factores a potenciar. Fuente: Elaborada por los autores
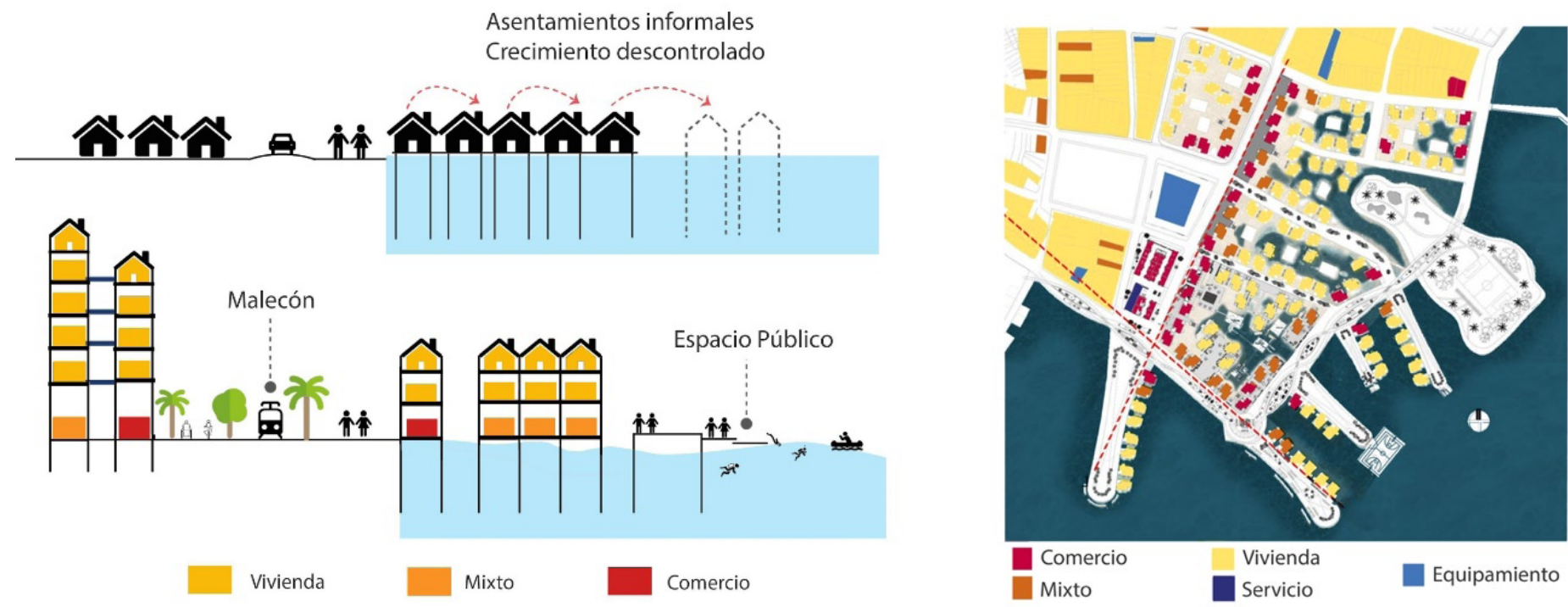

Figura 7. Altura y usos propuestos. Fuente: Elaborada por los autores.

\section{ESCALA LOCAL}

\section{Uso sostenible de los recursos}

Ante todo, se pretende reusar el suelo y la estructura urbana ya existentes con el fin de no generar intervenciones que traigan consigo más daño al territorio -en especial el crecimiento descontrolado hacia el mar y la deforestación de los manglares-, favoreciendo una estructura urbana más compacta y menos extendida (Urriza y Garriz, 2014). Seguidamente, debido a la importante relación con el mar que tienen los habitantes de Buenaventura, dentro del diseño urbano se contemplan las distintas actividades económicas que se dan a través de este cuerpo de agua, como: la pesca, una vía distinta a la terrestre para movilizarse entre las zonas aledañas y el área continental, además de su relación con las tradiciones de los lugareños y su connotación cultural. En tercer lugar, la implementación de nuevos mecanismos para suplir consumo energético se da en dos escalas. A escala macro, se pretende usar fuentes de energías renovables para los espacios públicos, además de la implementación del tranvía eléctrico como transporte público. A escala micro, se busca replicar el sistema fotovoltaico usado en el prototipo de vivienda, al igual que el sistema de recolección de aguas lluvias y los sensores inteligentes para controlar el consumo de cada unidad habitacional. Cabe agregar que, en ambas escalas existe la posibilidad de generar otras opciones de energía renovable, como la eólica, mareomotriz y undimotriz.

\section{Uso sostenible del espacio construido y las actividades urbanas}

En concordancia con el lema del equipo MINGA, "Comunidades sostenibles", se definieron tres factores a potenciar para el desarrollo del proyecto urbano en Buenaventura: la naturaleza, el turismo y el comercio (Figura 6). Mediante estas tres dimensiones se promueve la mixtura de usos en el proyecto, sin perder el propósito principal de desarrollar un modelo urbano de vivienda sostenible. De igual manera, están pensadas con el fin de incentivar los respectivos sectores de la economía y regenerar los espacios públicos de la ciudad, para potenciarla como un destino turístico y económico en Colombia, y balancear, consecuentemente, la calidad del sector residencial. Al mismo tiempo, se espera generar comunidades sostenibles, preservando los valores culturales de la población.

Así pues, la generación de distintos usos permite una mayor accesibilidad a los ciudadanos de acuerdo a las necesidades, reduciendo los tiempos y distancias de transporte. Es por 

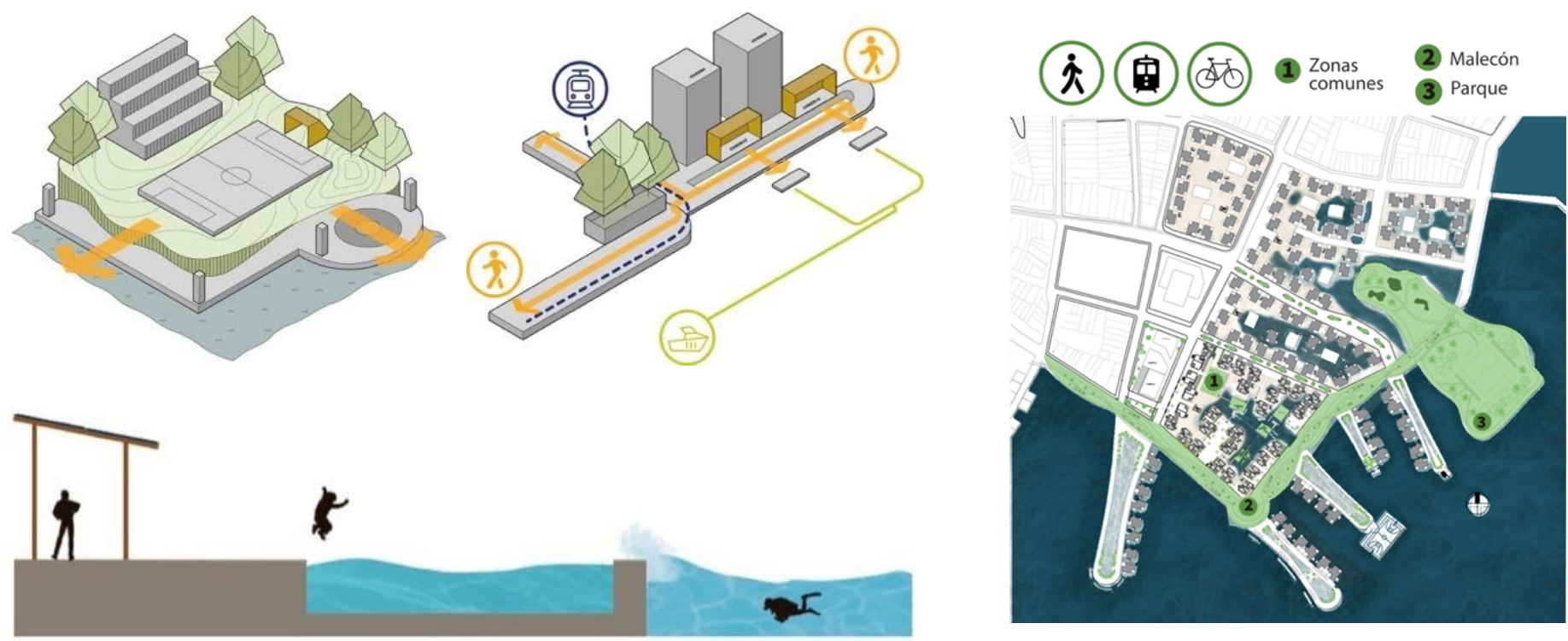

Figura 8. Diseño de espacios públicos / adaptabilidad e inundabilidad. Fuente: Elaborada por los autores.
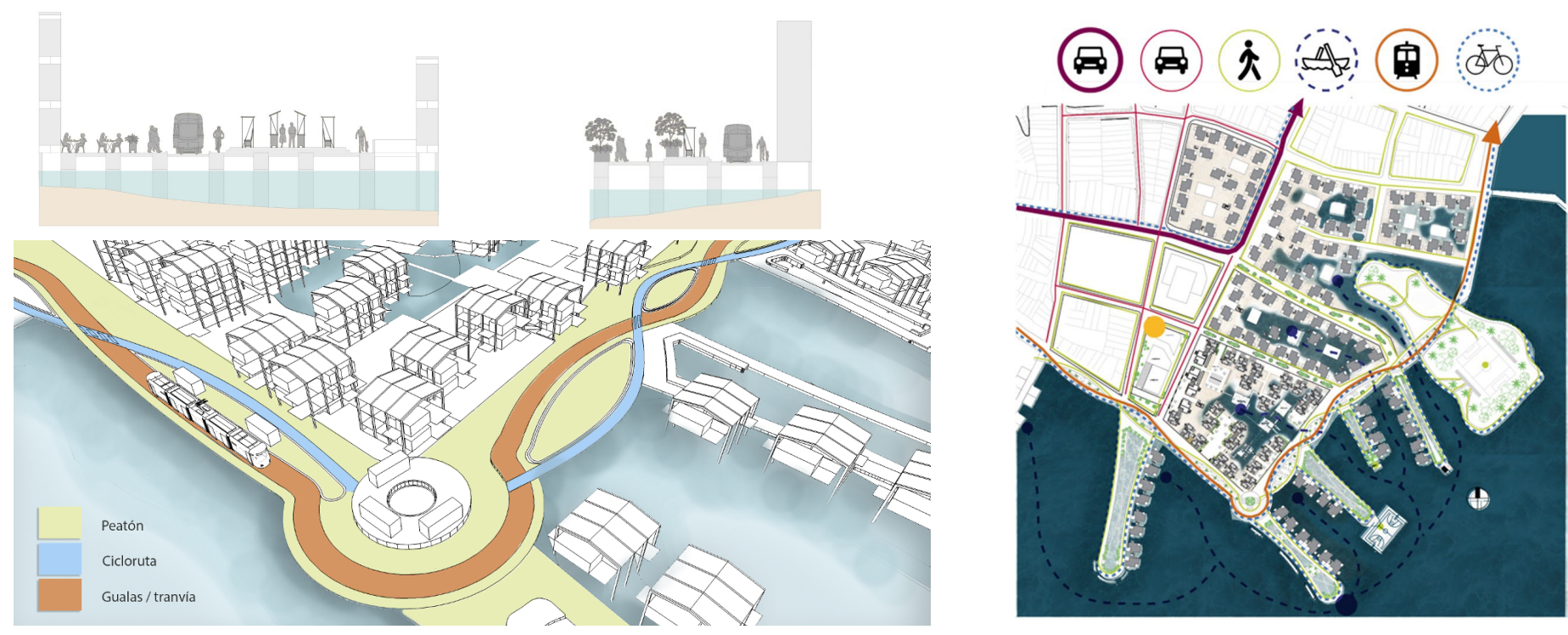

Figura 9. Propuesta de movilidad sostenible / Malecón. Fuente: Elaborada por los autores.

esto que, en la planta pública del proyecto, se propone la disposición de unidades de comercio que respondan a las vías principales, tanto vehiculares como peatonales (en las que se incluye el malecón), y conserven la privacidad de la vivienda y sus áreas comunes al interior de las manzanas. En la misma línea, ya que la densificación en altura permite una mayor capacidad en cuanto a número de habitantes en una menor porción de suelo, se decidió realizar bloques en altura que no superaran los 6 pisos ni fueran menores a 3 pisos. De esa forma, los edificios resultan dispuestos de manera tal que su altura disminuye conforme se acercan al mar (Figura 7).

Espacio público efectivo y desarrollo de equipamientos La deficiencia y carencia de espacio público en Buenaventura muestran la necesidad de diseñar espacios públicos efectivos que brinden a los habitantes más lugares de calidad, de mayor envergadura, más accesibles y mejor diseñados. Se propone, entonces, el uso de los predios no construidos, con el fin de generar allí zonas públicas equipadas con juegos para niños, espacios de actividad física $y$, en la medida de lo posible, focos verdes para la ciudad. Estas zonas son diseñadas para ser resilientes ante una posible inundación, es decir, para que se adapten a los cambios de marea y así sea posible mitigar el impacto de posibles desastres naturales, cosa que también pretende el malecón (Figura 8).

\section{Transporte sostenible y accesibilidad}

Para mejorar y facilitar la conexión entre la isla de Cascajal y la porción continental de la ciudad, se diseñó el malecón, en el que se planea converjan distintos medios de transporte limpios (Figura 9). El diseño se despliega a partir de una sola superficie, demarcando cada zona para su respectivo 

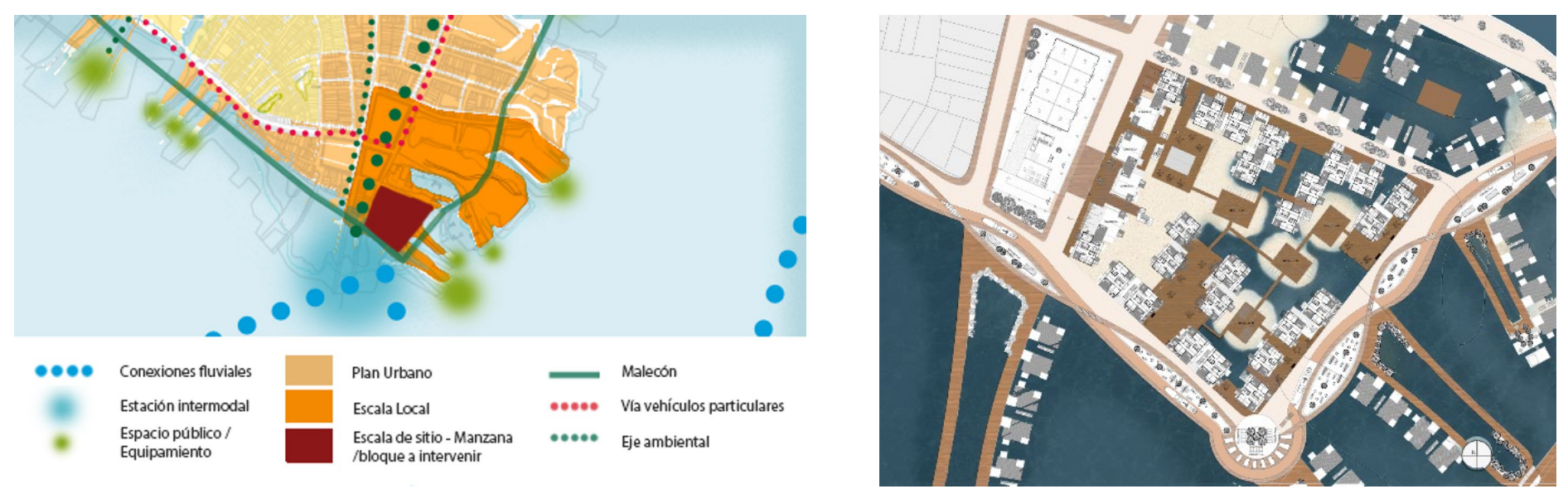

Figura 10. Localización y planta de bloque diseñado a escala de sitio. Fuente: Elaborada por los autores.

usuario, con el objetivo de democratizar el espacio y fomentar una cultura en la que se privilegie al peatón, dando también lugar a demás medios de transporte. En un inicio, una vía será destinada para las tradicionales gualas, el sistema de transporte público informal más usado en la isla, haciéndolo formal y procurando su transición futura hacia el tranvía como sistema de transporte limpio. Conjuntamente, se asignará una vía para la cicloruta y otra gran porción para el peatón, esta última contigua al mar. Como una solución adicional, a partir del entendimiento de los distintos medios de transporte de Buenaventura, se incluyó una vía paralela al malecón, la cual atravesaría también toda la isla conectándola con el continente y en la que se daría el flujo de automóviles y motos.

\section{ESCALA DE SITIO}

Para el proyecto urbano, el concurso SDLAC 2019 propuso el desarrollo a profundidad de un bloque o manzana en mayor detalle. Así, el equipo MINGA escogió un bloque o manzana al sur de la isla de Cascajal (Figura 10), en la comuna 3, zona en la que convergen distintas actividades y la que permitirá la conexión de la isla con la porción continental. Se resaltó la importancia de entender la manera cómo se concibe la ciudad para sus habitantes, las características de desarrollo urbano y sus formas de vida, derivado de lo cual se plantearon los siguientes objetivos:

- Resolver la alta demanda de vivienda efectiva por la creciente densidad poblacional en la isla, comenzando por las comunas 3 y 4 , en las que existe mayor cantidad de viviendas construidas de manera informal, a fin de que se densifique en altura, liberando mayor espacio común y público en la primera planta, para lo cual se propone una densidad de mínimo 120 viviendas por hectárea.

- Lograr un balance entre la densidad de habitantes y el espacio público efectivo, mejorando y manteniendo la vida en comunidad.

- Propiciar las relaciones ya existentes con el mar, de modo que no se pierdan a pesar del cambio respecto a la forma de construcción en la vivienda.
- Diseñar un modelo de vivienda sostenible, el cual considere la posibilidad de albergar hasta ocho personas en dos módulos de vivienda independientes, respondiendo a la forma de vida de las familias bonaverenses en la que es común encontrar más de un núcleo familiar por unidad de vivienda. Además, debe ser más factible económicamente, sin sobrepasar los topes de la política de subsidio a la vivienda de interés social de Colombia, lo que vuelve el proyecto accesible a la población objetivo.

- Incluir zonas comunes para la integración de la comunidad, en las que se pueda permanecer mientras la marea sea baja, sean inundables cuando suba la marea y sean un medio de conexión y transporte con el mar.

- Las áreas comunes de circulación en las edificaciones serán puntos para propiciar el encuentro como una "extensión" de las zonas privadas de estancia, que favorezcan la vida en comunidad.

- Hacer uso de energías renovables.

- Realizar un diseño estructural acorde con una tipología de edificación resiliente, que permita contrarrestar los posibles efectos del cambio climático y el consecuente crecimiento del nivel del mar (Figura 11).

- Disponer algunas unidades de vivienda en la planta pública para la adaptación de pequeños locales comerciales; práctica muy usada en este sector de la ciudad.

\section{SISTEMA CONSTRUCTIVO: REINTERPRETACIÓN DE LA CONSTRUCCIÓN TRADICIONAL PALAFÍTICA}

Se efectuó una reinterpretación de la tipología de vivienda palafítica, con un sistema estructural novedoso en Colombia, hecho a partir pórticos de madera laminada encolada, en reemplazo de la madera de manglar utilizada tradicionalmente, que permite la construcción de edificios multifamiliares de hasta seis pisos. Con ello, se reduce el impacto por deforestación de estos ecosistemas. Por otra parte, se pretende minimizar el riesgo de inundación por mareas en el clima actual y futuro y frenar el crecimiento informal de la isla mediante la densificación en altura de 

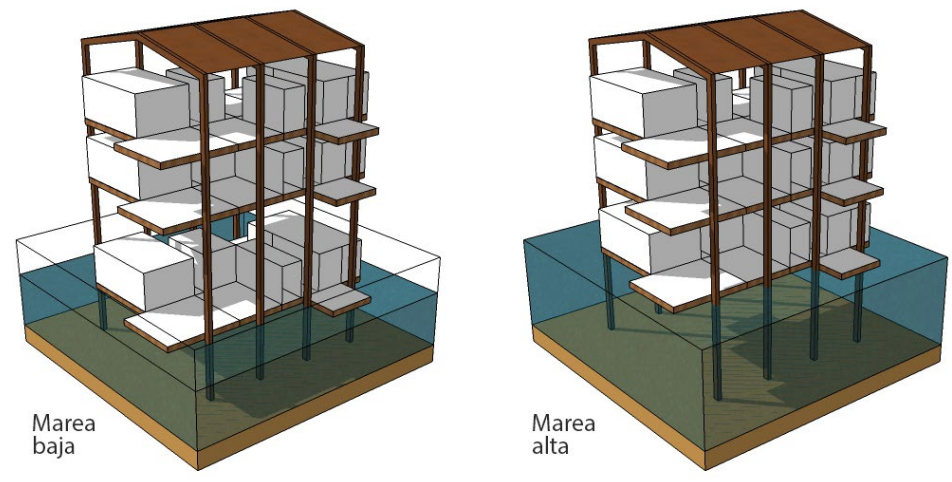

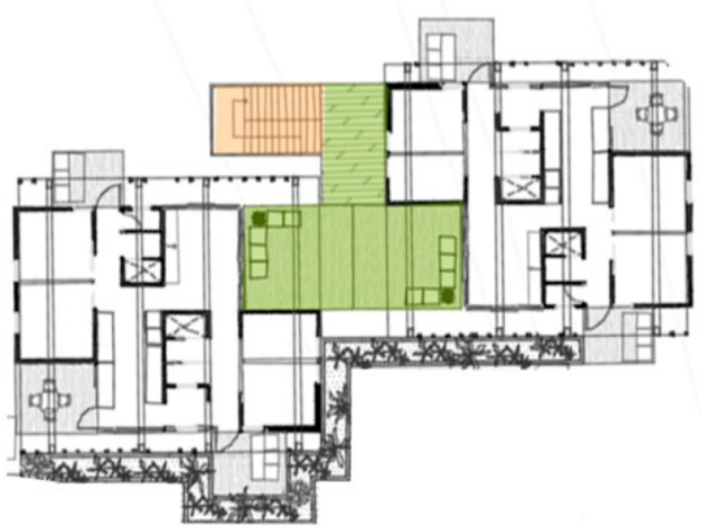

SECTION A-A

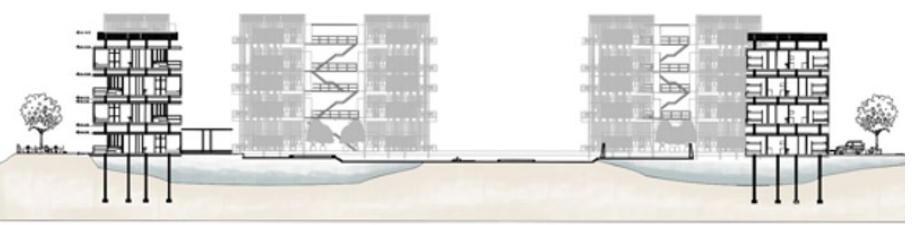

SECTION B-B

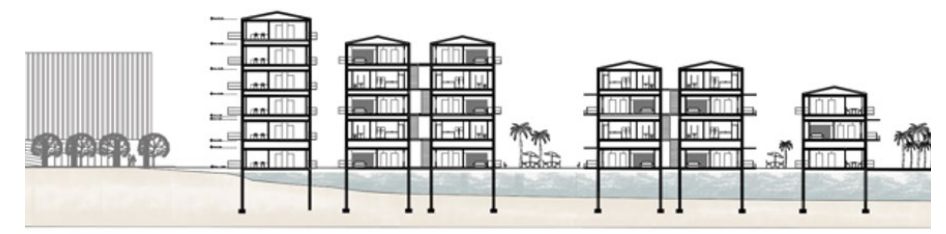

Figura 11. Esquema de flotabilidad, planta tipo y secciones del conjunto de viviendas. Fuente: Elaborada por los autores.

una zona urbana, aunado al diseño de equipamientos y espacio público hacia el borde de la isla.

\section{VIABILIDAD: POLIITICA DE SUBSIDIO Y FINANCIACIÓN}

La política de VIS (Vivienda de Interés Social) en Colombia tiene un sistema de subsidio a la demanda. Esta política busca incrementar la demanda efectiva de vivienda de interés social mediante subsidios a los beneficiarios, los que se complementan con créditos blandos y ahorro para que las familias con menos recursos puedan acceder a una vivienda propia. El valor otorgado a las familias beneficiarias, depende del rango de ingresos familiares y del costo total de la vivienda. Para promover el desarrollo de proyectos en áreas urbanas con tratamiento de renovación, el gobierno nacional amplió el tope de la vivienda de interés social objeto de subsidio hasta 175 SMMLV (Salarios Mínimo Mensual Legal Vigente) que equivalen, para el año 2020, a 153.615.525 COP (pesos colombianos), aproximadamente 41.794 USD (dólares estadounidenses) al 27 de julio de 2020.

El proyecto multifamiliar MINGA para la isla de Cascajal integra viviendas en bloques de edificios de hasta 6 pisos de altura, alcanzando una densidad de 127 viviendas por hectárea, cada una con opción de albergar hasta 2 núcleos familiares (8 personas máximo), con altos estándares de accesibilidad y calidad de los espacios públicos y colectivos. Lo anterior, mediante sistemas de construcción y prefabricación industrializada, y el empleo de materiales locales. Para enmarcarse en este rango de precios, la iniciativa plantea estrategias que fomentan el mejoramiento de la calidad en las condiciones urbanas del área de intervención, con las siguientes condiciones:
- Promueve la densificación del área con un proyecto integral que garantiza la construcción de equipamientos, servicios complementarios y de espacio público.

- Garantiza la prestación adecuada y eficiente de los servicios públicos domiciliarios.

- Prevé un adecuado uso y manejo del ambiente y de los recursos naturales.

- Promueve la protección e integración de las áreas de conservación y protección ambiental, de acuerdo con lo definido en el POT.

- Articula la vivienda de interés social con la infraestructura para el sistema vial de transporte.

- Promueve y genera redes de movilidad accesibles para personas con discapacidad y dificultades de locomoción a partir de la eliminación de barreras físicas.

- Mejora los estándares cualitativos de espacio público, buscando incrementar la calidad, la dotación y el mejor aprovechamiento de los espacios públicos existentes.

- Garantiza los sistemas de equipamientos colectivos que suplen las necesidades de la nueva población que se incorpora a la zona.

- Incorpora determinantes de gestión y prevención del riesgo en un sector que el plan de ordenamiento territorial ha identificado como de riesgo mitigable alto o medio.

\section{CONCLUSIONES}

Con los resultados del proyecto MINGA, se demuestra la viabilidad de las estrategias propuestas para la renovación de los frentes marítimos en las ciudades costeras del 
trópico mediante viviendas de bajo costo en altura, con una densidad habitacional que usa adecuadamente la infraestructura urbana existente y logra frenar el crecimiento de las áreas urbanas de las ciudades, atendiendo una problemática muy sensible en las ciudades litorales de América Latina y el Caribe (Barragán y De Andrés, 2016). El proyecto logra altos estándares de sostenibilidad puesto que su costo de construcción permite que sea subsidiado dentro de la política de VIS en Colombia. Este tipo de vivienda sostenible genera un menor costo para los usuarios durante su vida útil, ya que reduce el valor de los servicios públicos, gracias al uso de energía solar fotovoltaica, el aprovechamiento de las aguas lluvias y el usufructo económico de la propiedad, disponiendo un módulo como local comercial o a partir del arriendo de este como segunda unidad de vivienda de la casa.

Para concluir, si bien la propuesta urbana se sitúa en la ciudad de Buenaventura, Colombia, y teniendo en cuenta sus condiciones medio ambientales, sociales y económicas, las estrategias proyectuales empleadas son adaptables a otros territorios con condiciones similares. Así, el modelo de renovación urbana es replicable en cualquier ciudad costera con clima cálido - húmedo tropical, siempre y cuando se consideren las diferencias en cuanto a las condiciones, adecuándose al contexto en el que se desee implantar: contemplar niveles de riesgo, relaciones con la estructura urbana del lugar, características de la población y políticas de vivienda de los gobiernos locales.

En suma, se demuestra la factibilidad de desarrollar renovaciones urbanas sostenibles y se releva la importancia de generar proyectos urbanos que respondan a las necesidades de las comunidades -entendiendo las relaciones que estas tienen con su entorno-, como también de buscar soluciones que comprendan las particularidades del territorio existentes.

\section{AGRADECIMIENTOS}

Se agradece a los miembros del equipo MINGA, estudiantes, profesores, colaboradores y voluntarios de la Pontificia Universidad Javeriana Cali, de la Universidad Federal de Santa Catarina y el Instituto Federal de Santa Catarina. También a los organizadores del Solar Decathlon Latin America \& Caribbean 2019.

\section{REFERENCIAS BIBLIOGRÁFICAS}

Alcaldía de Buenaventura (2001). Acuerdo 03 de 2001, Plan de Ordenamiento Territorial POT "Convivencia Pacífica desde la Diversidad", Buenaventura 2001.

Barragán, J. M. y De Andrés, M. (2016). Expansión urbana en las áreas litorales de América Latina y Caribe. Revista de Geografía Norte Grande, (64), 129-149.
Béné, C., Mehta, L., Mcgranahan, G., Cannon, T., Gupte, J. y Tanner, T., (2018). Resilience as a policy narrative: potentials and limits in the context of urban planning. Clim. Dev., 10(2), 116-133. DOI: https://doi.org/10.1080/17565529.2017.1301868

Cocuñame Ricardo, D. y Salcedo Hurtado, E. (2017). Evaluación del riesgo de inundación ante tsunami local en la isla de Cascajal, Pacífico Colombiano. Revista de geografía Norte Grande, (68), 185-219.

Departamento Administrativo Nacional de Estadística [DANE] (2005). Proyecciones de población municipales por área. En: Proyección municipios 2005 - 2020. Bogotá D.C.

Departamento Administrativo Nacional de Estadística [DANE] (2018). Necesidades Básicas Insatisfechas Comparación CG 2005 y CNPV 2018. En: Resultados Censo Nacional de Población y Vivienda 2018. Bogotá D.C.

Departamento Administrativo Nacional de Estadística [DANE] (2020). La información del DANE en la toma de decisiones de los municipios del país. Buenaventura, Valle del Cauca. Recuperado de https://www.dane.gov.co/files/investigaciones/planesdesarrollo-territorial/100320-Info-Alcaldia-Buenaventura.pdf

Hernández Araque, M.J. (2016). Urbanismo participativo. Construcción social del espacio urbano. Rev. Arquit., 18(1), 6-17. DOI: https://doi.org/10.14718/revarq.2016.18.1.2

Hernández-Guerrero, J., Vieyra-Medrano, A. y Mendoza, M.E., (2012). Adaptation strategies in communities under precarious housing: Flooding risks in the peri-urban sector of the city of Morelia, Michoacán, México. Appl. Geogr., 34, 669-679. DOI: https://doi.org/10.1016/j.apgeog.2012.04.010

Hernantes, J., Maraña, P., Giménez, R., Sarriegi, J.M. y Labaka, L. (2019). Towards resilient cities: A maturity model for operationalizing resilience. Cities, 84, 96-103. https://doi. org/10.1016/j.cities.2018.07.010

Herrera-Limones, R., León-Rodríguez, Á.L., López-Escamilla, Á. (2019). Solar Decathlon Latin America and Caribbean: Comfort and the balance between passive and active design. Sustainability, 11(13). DOI: https://doi.org/10.3390/su11133498

Herrera-Limones, R., Rey-Pérez, J., Hernández-Valencia, M. y RoaFernández, J. (2020). Student competitions as a learning method with a sustainable focus in higher education: The University of Seville "Aura Projects" in the "Solar Decathlon 2019". Sustainability, 12(4). DOI: https://doi.org/10.3390/su12041634

Jin, R., Yang, T., Piroozfar, P., Kang, B.G., Wanatowski, D., Hancock, C.M. y Tang, L. (2018). Project-based pedagogy in interdisciplinary building design adopting BIM. Eng. Constr. Archit. Manag., 25(10), 1376-1397. DOl: https://doi.org/10.1108/ ECAM-07-2017-0119

Kos, J.R. y De Souza, B.M. (2014). Educating home users through a solar house: The Ekó House experience. Energy Build., 83, 181185. DOI: https://doi.org/10.1016/j.enbuild.2014.03.080

Long, J. y Rice, J.L., 2019. From sustainable urbanism to climate urbanism. Urban Stud., 56(5), 992-1008. DOI: https://doi. org/10.1177/0042098018770846 
Martínez, A., García, H., Ramírez, C., Ramírez, D., Restrepo, J., Ortega, P., ... Rocha, L. A. (2013). Hacia un desarrollo integral de la ciudad de Buenaventura y su área de influencia. Bogotá: Fedesarrollo-CERAC.

Meerow, S., Newell, J.P. y Stults, M. (2016). Defining urban resilience: A review. Landsc. Urban Plan., 147, 38-49. DOI: https://doi.org/10.1016/j.landurbplan.2015.11.011

Musango, J K., Currie, P., Smit, S. y Kovacic, Z. (2020). Urban metabolism of the informal city: Probing and measuring the 'unmeasurable' to monitor Sustainable Development Goal 11 indicators. Ecol. Indic., 119. DOI: https://doi.org/10.1016/j. ecolind.2020.106746

Neumann, B., Vafeidis, A.T., Zimmermann, J. y Nicholls, R.J., 2015. Future coastal population growth and exposure to sea-level rise and coastal flooding - A global assessment. PLoS One, 10(6). DOI: https://doi.org/10.1371/journal.pone.0118571

Nicholls, R.J., Hanson, S.E., Lowe, J.A., Warrick, R.A., Lu, X. y Long, A.J., 2014. Sea-level scenarios for evaluating coastal impacts. Wiley Interdiscip. Rev. Clim. Chang., 5(1), 129-150. DOI: https://doi.org/10.1002/wcc.253

Osuna-Motta, I. (2018). Aprender haciendo: La Experiencia De Aprendizaje En Construcción Sostenible Del Solar Decathlon. En Cortés, M. et al. (Eds.), Sustentabilidad y tecnología: herramientas para la gestión segura y eficiente del hábitat (Hábitat Sustentable Contemporáneo) (pp. 119-144). https://doi. org/10.2307/j.ctvjhzpn5.8

Rafferty J. (2009). Köppen climate classification. Wet equatorial climate. En Tropical monsoon and trade-wind littoral climate. Encyclopedia Britannica. Recuperado de https://www.britannica. com/science/Koppen-climate-classification

Schoening, M. (2014). The crisis in Buenaventura: disappearances, dismemberment, and displacement in Colombia's main Pacific Port. Human Rights Watch.

Solar Decathlon Latin America \& Caribbean [SDLAC] (2019). Rules Adjusted Version. Recuperado de https://solardecathlonlac.com

Urriza, G. y Garriz, E. (2014). ¿Expansión urbana o desarrollo compacto? Estado de situación en una ciudad intermedia: Bahía Blanca, Argentina. Revista Universitaria de Geografía, 23(2), 97123.

Vergel Tovar, E. (2010). Asentamientos precarios Una aproximación para su mejoramiento integral y prevención. Dearq, (6), 64-81. DOI: https://doi.org/10.18389/dearq6.2010.08

Wardekker, J.A., De Jong, A., Knoop, J.M. y Van Der Sluijs, J.P. (2010). Operationalising a resilience approach to adapting an urban delta to uncertain climate changes. Technol. Forecast. Soc. Change, 77(6), 987-998. https://doi.org/10.1016/j. techfore.2009.11.005

World Health Organization [WHO] (2016). Urban green spaces and health. Copenhagen: WHO Regional Office for Europe. 